\title{
A NEW AUTOMATED SAMPLE OF COMPACT GROUPS OF GALAXIES
}

\author{
A. IOVINO AND E. TASSI \\ Oss. Astron. di Brera, Milano, Italy \\ C. MENDES DE OLIVEIRA \\ USP, Sao Paolo, Brazil \\ P. HICKSON \\ UBC, Vancouver, Canada \\ AND \\ H. MACGILLIVRAY \\ ROE, Edinburgh, Great Britain
}

Compact Groups (CGs) of galaxies represent an extreme class of objects. They typically contain 4-8 galaxies with high space density (as in the centers of rich clusters) but with low velocity dispersions, being an excellent laboratory for the study of galaxy interactions and their effects. Up to now no unbiassed sample of CGs was available in the literature, and therefore several of the classical problems and paradoxes involving CGs could not be properly addressed.

We exploited the availability of large digitized galaxy catalogs to develop an algorithm for the detection of CGs, implementing clearly defined and rigidly applied selection criteria. The new algorithm is optimized for work also at fainter magnitudes, where both incompleteness and contamination become serious problems. We applied the new algorithm to an area of 4500 sq. deg. in the Southern Sky, obtaining $\sim 300$ candidate CGs up to magnitude $b_{\mathrm{j}} \sim 16.0$ for the brightest galaxy of the group.

Using the ESO 1.5m telescope at LaSilla, we measured redshifts for all the members of 60 candidate CGs, defining a brighter subsample (up to $b_{\mathrm{j}} \sim 14.5$ for the brightest galaxy of the group). Assuming $1000 \mathrm{~km} \mathrm{~s}^{-1}$ as the velocity cut-off for group membership, 48 candidates have three or more concordant members, corresponding to an increase of $\sim 4$ times in surface density with respect to Hickson's sample of CGs! 